Article

\title{
Synthesis and Insecticidal Activities of New Ester-Derivatives of Celangulin-V
}

\author{
Jiwen Zhang, Zhan Hu, Shenkun Li and Wenjun Wu * \\ Institute of Pesticide Science, Northwest A\&F University, Yangling, \\ Shaanxi 712100, China; E-Mails: nwzjw@yahoo.com.cn (J.Z.); huzhan123cn@yahoo.cn (Z.H.); \\ saintkun001@yeah.net (S.L.)
}

* Author to whom correspondence should be addressed; E-Mail: wuwenjun@nwsuaf.edu.cn; Tel.: +86-29-8709-2191; Fax: +86-29-8709-3987.

Received: 17 August 2011; in revised form: 28 October 2011 / Accepted: 15 December 2011 / Published: 20 December 2011

\begin{abstract}
In order to develop new biorational pesticides, ten new 6-substituted ester derivatives of Celangulin-V were designed and synthesized. The structures of the new derivatives were confirmed by IR, ${ }^{1} \mathrm{H}-\mathrm{NMR},{ }^{13} \mathrm{C}-\mathrm{NMR}$ and ESI-MS spectral analysis. Insecticidal activities of these compounds were tested against the third-instar larvae of Mythimna separata. Two derivatives $(\mathbf{1 . 1}, \mathbf{1 . 2})$ showed higher insecticidal activities than Celangulin- $\mathrm{V}$, with mortality of $75.0 \%$ and $83.3 \%$, respectively. While four compounds $(1.3,1.4,1.7,1.8)$ denoted lower insecticidal activities, the others $(1.5,1.6,1.9,1.10)$ revealed no activities at a concentration of $10 \mathrm{mg} \cdot \mathrm{mL}^{-1}$. The results suggest that C-6 substitutions of Celangulin- $\mathrm{V}$ are very important in determining the insecticidal activities of its ester-derivatives. That the acetyl (1.1) and propionyl (1.2) derivatives possessed much higher insecticidal activities than Celangulin- $\mathrm{V}$ itself supported the view that Celangulin- $\mathrm{V}$ has the potential to be a lead structure of semi-synthetic green insecticides.
\end{abstract}

Keywords: Celastraceae; dihydroagarofuran; biorational pesticides

\section{Introduction}

Plants have been a major source of chemical structure models for insecticides. Several important classes of synthetic insecticides have ancestors in nature. As a result of our program of screening 
insecticidal activity constituted from plants of China, some sesquiterpene polyol esters with insecticidal activity were isolated [1-4]. Celangulin-V (Figure 1), a $\beta$-dihydroagrofuran sesquiterpene polyol ester, from the root bark of Chinese bittersweet, Celastrus angulatus Max [5,6], showed prominent stomach toxic effects against Mythimna separate acting on mid-gut tissue with a special mechanism of toxicology and suggested it to be a new lead compound for a botanical insecticide [7].

Figure 1. Structure of Celangulin-V.

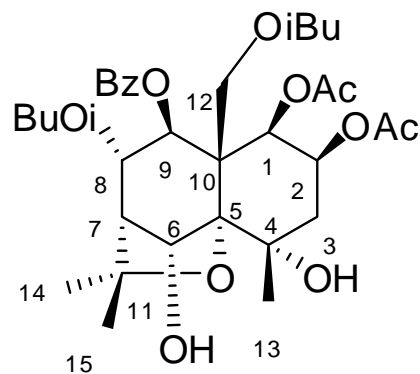

With the objective to search for more potent compounds than Celangulin- $\mathrm{V}$ and to clarify the potential structural factors needed for the biological activity of Celangulin-V analogues, ten new C-6 substituted Celangulin- $\mathrm{V}$ ester analogues were designed and synthesized. The biological activities of these compounds were tested against the third-instar larvae of Mythimna separata.

Like its C-6 ether analogues [8], the insecticidal activities of Celangulin-V C-6 esters varied with the different C-6 substitution. Concurrently, the symptoms of the tested larvae of Mythimna separata. were characterized in the same way as in our previous reports[3,4,6-8]. In this paper, the synthesis, characterization and insecticidal activity of new C- 6 substituted Celangulin- $\mathrm{V}$ esters is described.

\section{Results and Discussion}

\subsection{Synthesis of Celangulin-V Derivatives}

As shown in Scheme 1, ten new 6-acyloxy derivatives (1.1-1.10) of Celangulin-V were successfully prepared. Compound 1 reacted with anhydrides in dried pyridine at room temperature to give Compounds 1.1, 1.2, 1.3 and 1.7. Compounds 1.4, 1.5, 1.6, 1.8, 1.9 and 1.10 were obtained from ordinary esterifications of $\mathbf{1}$ with carboxylic acids by using N,N-dicyclohexylcarbodiimide (DCC) as a condensation agent with a catalytic amount of 4-dimethylaminopyridine (DMAP) at room temperature. The structures of all the target compounds were well characterized by ${ }^{1} \mathrm{H}-\mathrm{NMR},{ }^{13} \mathrm{C}-\mathrm{NMR}$, ESI-MS, IR, and MP (see Tables 1-3). 
Scheme 1. Synthetic route of target compounds. N,N-dicyclohexylcarbodiimide (DCC),4-dimethylaminopyridine (DMAP), dichloromethane DCM and pyridine (pyr).

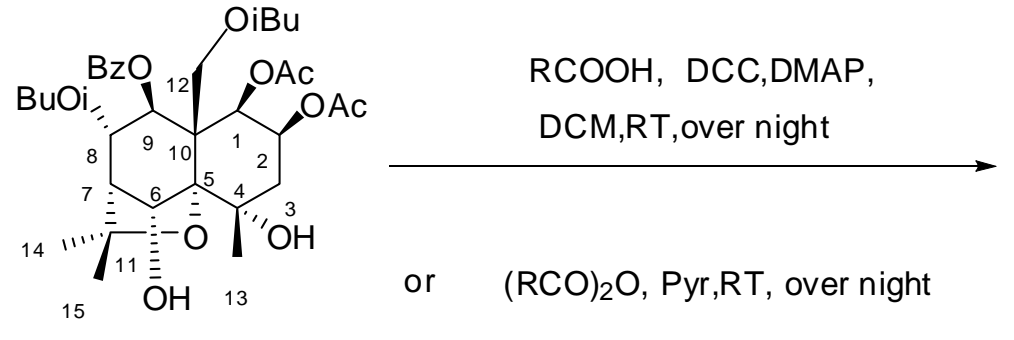

Celangulin- $\mathrm{V}$

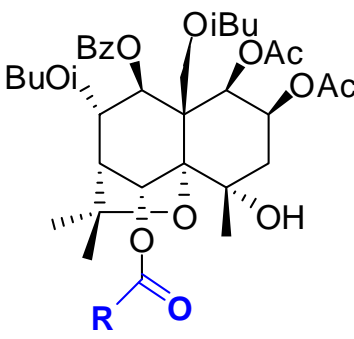

1.1-1.10
$1.2-\xi-\mathrm{CH}_{2} \mathrm{CH}_{3}$

$1.4-$ - $\mathrm{CH}_{2}\left(\mathrm{CH}_{2}\right)_{2} \mathrm{CH}_{3}$

$1.6-\mathrm{CH}_{2}\left(\mathrm{CH}_{2}\right)_{4} \mathrm{CH}_{3}$

$1.8-\left\{-\mathrm{CH}\left(\mathrm{CH}_{2} \mathrm{CH}_{3}\right)_{2}\right.$

1.10

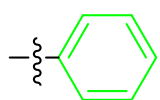

Table 1. Experimental Data of Celangulin-V and its derivatives.

\begin{tabular}{ccccc}
\hline Compounds & $\begin{array}{c}\text { Molecular } \\
\text { Formula }\end{array}$ & $\mathbf{M . P}^{\circ} \mathbf{C}$ & $\begin{array}{c}\text { ESI-MS } \\
\mathbf{( M + N a )}\end{array}$ & $\begin{array}{c}\text { Optical Rotation } \\
\left.[\boldsymbol{\alpha}]^{\circ}, \mathbf{( M e O H , ~}=\mathbf{m g . 1 0 0} \mathbf{~ m L}^{-\mathbf{1}}\right)\end{array}$ \\
\hline Celangulin-V & $\mathrm{C}_{34} \mathrm{H}_{46} \mathrm{O}_{13}$ & $198-200$ & 685 & $-12.2,(10.8)$ \\
$\mathbf{1 . 1}$ & $\mathrm{C}_{36} \mathrm{H}_{48} \mathrm{O}_{14}$ & $214-216$ & 727 & $-10.6,(16.0)$ \\
$\mathbf{1 . 2}$ & $\mathrm{C}_{37} \mathrm{H}_{50} \mathrm{O}_{14}$ & $94-96$ & 741 & $-10.1,(15.0)$ \\
$\mathbf{1 . 3}$ & $\mathrm{C}_{38} \mathrm{H}_{52} \mathrm{O}_{14}$ & $114-116$ & 755 & $-10.3,(18.5)$ \\
$\mathbf{1 . 4}$ & $\mathrm{C}_{39} \mathrm{H}_{54} \mathrm{O}_{14}$ & $102-104$ & 769 & $-33.0,(18.0)$ \\
$\mathbf{1 . 5}$ & $\mathrm{C}_{40} \mathrm{H}_{56} \mathrm{O}_{14}$ & $64-66$ & 783 & $-15.2,(15.0)$ \\
$\mathbf{1 . 6}$ & $\mathrm{C}_{41} \mathrm{H}_{58} \mathrm{O}_{14}$ & colorless oils & 797 & $-26.4,(19.0)$ \\
$\mathbf{1 . 7}$ & $\mathrm{C}_{38} \mathrm{H}_{52} \mathrm{O}_{14}$ & $98-100$ & 755 & $-39.6,(17.0)$ \\
$\mathbf{1 . 8}$ & $\mathrm{C}_{40} \mathrm{H}_{56} \mathrm{O}_{14}$ & $112-114$ & 783 & $-95.8,(11.5)$ \\
$\mathbf{1 . 9}$ & $\mathrm{C}_{43} \mathrm{H}_{54} \mathrm{O}_{14}$ & $124-126$ & 817 & $-29.7,(12.5)$ \\
$\mathbf{1 . 1 0}$ & $\mathrm{C}_{41} \mathrm{H}_{06} \mathrm{O}_{14}$ & $96-98$ & 789 & $-51.7,(13.5)$ \\
\hline
\end{tabular}


Table 2. ${ }^{13} \mathrm{C}$-NMR Spectral Data $[125 \mathrm{MHz}, \delta(\mathrm{ppm})]$ for compounds $\mathbf{1}$ and $\mathbf{1 . 1 - 1 0}$ in $\mathrm{CDCl}_{3}$.

\begin{tabular}{cccccccccccc}
\hline No. & $\mathbf{1}$ & $\mathbf{1 . 1}$ & $\mathbf{1 . 2}$ & $\mathbf{1 . 3}$ & $\mathbf{1 . 4}$ & $\mathbf{1 . 5}$ & $\mathbf{1 . 6}$ & $\mathbf{1 . 7}$ & $\mathbf{1 . 8}$ & $\mathbf{1 . 9}$ & $\mathbf{1 . 1 0}$ \\
\hline 1 & 75.17 & 75.20 & 75.22 & 75.22 & 75.23 & 75.22 & 75.23 & 75.23 & 75.25 & 75.19 & 75.20 \\
2 & 67.44 & 67.69 & 67.71 & 67.70 & 67.72 & 67.71 & 67.72 & 67.71 & 67.72 & 67.68 & 67.74 \\
3 & 41.25 & 42.01 & 41.98 & 41.94 & 41.94 & 41.93 & 41.94 & 41.99 & 41.79 & 41.99 & 42.51 \\
4 & 72.23 & 69.61 & 69.64 & 69.63 & 69.63 & 69.63 & 69.65 & 69.64 & 69.64 & 69.59 & 69.81 \\
5 & 91.56 & 92.09 & 92.11 & 92.08 & 92.08 & 92.08 & 92.08 & 92.17 & 92.04 & 92.08 & 91.94 \\
6 & 77.04 & 75.60 & 75.58 & 75.59 & 75.60 & 75.60 & 75.60 & 75.61 & 75.69 & 75.63 & 76.43 \\
7 & 53.69 & 52.31 & 52.36 & 52.40 & 52.39 & 52.39 & 52.39 & 52.43 & 52.62 & 52.31 & 52.31 \\
8 & 73.86 & 75.19 & 73.57 & 73.54 & 73.55 & 73.54 & 73.55 & 73.60 & 73.48 & 73.51 & 73.39 \\
9 & 75.45 & 75.54 & 75.49 & 75.46 & 75.49 & 75.49 & 75.50 & 75.42 & 75.69 & 75.53 & 75.70 \\
10 & 50.71 & 51.46 & 51.47 & 51.47 & 51.48 & 51.47 & 51.48 & 51.49 & 51.54 & 51.45 & 51.57 \\
11 & 84.61 & 84.34 & 84.31 & 84.29 & 84.29 & 84.28 & 84.29 & 84.29 & 84.21 & 84.27 & 84.44 \\
12 & 61.82 & 61.79 & 61.80 & 61.79 & 61.80 & 61.80 & 61.81 & 61.79 & 61.79 & 61.79 & 61.87 \\
13 & 24.32 & 24.85 & 24.93 & 24.93 & 24.96 & 24.97 & 24.98 & 24.92 & 25.13 & 24.76 & 24.57 \\
14 & 26.45 & 25.89 & 25.90 & 25.89 & 25.90 & 25.90 & 25.91 & 25.89 & 25.87 & 25.86 & 25.89 \\
15 & 30.15 & 29.72 & 29.72 & 29.73 & 29.74 & 29.74 & 29.75 & 29.75 & 29.83 & 29.66 & 29.81 \\
\hline
\end{tabular}

Table 3. ${ }^{1} \mathrm{H}-\mathrm{NMR}$ Spectral Data $\left[500 \mathrm{MHz}, \delta_{\mathrm{H}}(\mathrm{J}\right.$ in $\left.\mathrm{Hz})\right]$ of compounds 1 and 1.1-10 in $\mathrm{CDCl}_{3}$.

\begin{tabular}{|c|c|c|c|c|c|c|c|c|c|c|c|}
\hline No. & 1 & 1.1 & 1.2 & 1.3 & 1.4 & 1.5 & 1.6 & 1.7 & 1.8 & 1.9 & 1.10 \\
\hline & 5.49 & 5.48 & 5.48 & 5.48 & 5.48 & 5.48 & 5.48 & 5.48 & 5.49 & 5.46 & 5.52 \\
\hline 1 & $\begin{array}{c}(\mathrm{d}, 1 \mathrm{H}, \\
3.5)\end{array}$ & $\begin{array}{c}(\mathrm{d}, 1 \mathrm{H}, \\
3.5)\end{array}$ & $\begin{array}{c}(\mathrm{d}, 1 \mathrm{H}, \\
3.5)\end{array}$ & $\begin{array}{c}(\mathrm{d}, 1 \mathrm{H}, \\
3.5)\end{array}$ & $\begin{array}{c}(\mathrm{d}, 1 \mathrm{H}, \\
3.5)\end{array}$ & $\begin{array}{c}(\mathrm{d}, 1 \mathrm{H}, \\
3.5)\end{array}$ & $\begin{array}{c}(\mathrm{d}, 1 \mathrm{H}, \\
3.5)\end{array}$ & $\begin{array}{c}(\mathrm{d}, 1 \mathrm{H}, \\
3.5)\end{array}$ & $\begin{array}{c}(\mathrm{d}, 1 \mathrm{H}, \\
3.5)\end{array}$ & $\begin{array}{c}(\mathrm{d}, 1 \mathrm{H}, \\
3.5)\end{array}$ & $\begin{array}{c}(\mathrm{d}, 1 \mathrm{H}, \\
3.5)\end{array}$ \\
\hline & 5.39 & 5.36 & 5.36 & 5.36 & 5.36 & 5.36 & 5.36 & 5.36 & 5.37 & 5.35 & 5.39 \\
\hline 2 & $(\mathrm{~m}, 1 \mathrm{H})$ & $(\mathrm{m}, 1 \mathrm{H})$ & $(\mathrm{m}, 1 \mathrm{H})$ & $(\mathrm{m}, 1 \mathrm{H})$ & $(\mathrm{m}, 1 \mathrm{H})$ & $(\mathrm{m}, 1 \mathrm{H})$ & $(\mathrm{m}, 1 \mathrm{H})$ & $(\mathrm{m}, 1 \mathrm{H})$ & $(\mathrm{m}, 1 \mathrm{H})$ & $(\mathrm{m}, 1 \mathrm{H})$ & $(\mathrm{m}, 1 \mathrm{H})$ \\
\hline & 2.09 & 2.11 & 2.11 & 2.12 & 2.14 & 2.12 & 2.12 & 2.13 & 2.12 & 2.11 & 2.17 \\
\hline 3 & $\begin{array}{c}(\mathrm{m}, 1 \mathrm{H}) \\
2.03 \\
(\mathrm{~m}, 1 \mathrm{H})\end{array}$ & $\begin{array}{c}(\mathrm{m}, 1 \mathrm{H}), \\
1.97 \\
(\mathrm{~m}, 1 \mathrm{H})\end{array}$ & $\begin{array}{c}(\mathrm{m}, 1 \mathrm{H}), \\
1.96 \\
(\mathrm{~m}, 1 \mathrm{H})\end{array}$ & $\begin{array}{c}(\mathrm{m}, 1 \mathrm{H}), \\
2.07 \\
(\mathrm{~m}, 1 \mathrm{H})\end{array}$ & $\begin{array}{c}(\mathrm{m}, 1 \mathrm{H}) \\
2.09 \\
(\mathrm{~m}, 1 \mathrm{H})\end{array}$ & $\begin{array}{c}(\mathrm{m}, 1 \mathrm{H}) \\
1.95 \\
(\mathrm{~m}, 1 \mathrm{H})\end{array}$ & $\begin{array}{c}(\mathrm{m}, 1 \mathrm{H}) \\
1.95 \\
(\mathrm{~m}, 1 \mathrm{H})\end{array}$ & $\begin{array}{c}(\mathrm{m}, 1 \mathrm{H}) \\
1.95 \\
(\mathrm{~m}, 1 \mathrm{H})\end{array}$ & $\begin{array}{c}(\mathrm{m}, 1 \mathrm{H}) \\
1.95 \\
(\mathrm{~m}, 1 \mathrm{H})\end{array}$ & $\begin{array}{c}(\mathrm{m}, 1 \mathrm{H}) \\
1.95 \\
(\mathrm{~m}, 1 \mathrm{H})\end{array}$ & $\begin{array}{c}(\mathrm{m}, 1 \mathrm{H}), \\
2.02 \\
(\mathrm{~m}, 1 \mathrm{H})\end{array}$ \\
\hline 6 & $\begin{array}{c}5.22(\mathrm{~s} \\
1 \mathrm{H})\end{array}$ & $\begin{array}{c}6.48(\mathrm{~s} \\
1 \mathrm{H})\end{array}$ & $\begin{array}{c}6.49(\mathrm{~s} \\
1 \mathrm{H})\end{array}$ & $\begin{array}{c}6.49(\mathrm{~s} \\
1 \mathrm{H})\end{array}$ & $\begin{array}{c}6.48(\mathrm{~s} \\
1 \mathrm{H})\end{array}$ & $\begin{array}{c}6.48(\mathrm{~s} \\
1 \mathrm{H})\end{array}$ & $\begin{array}{c}6.48(\mathrm{~s} \\
1 \mathrm{H})\end{array}$ & $\begin{array}{c}6.49(\mathrm{~s} \\
1 \mathrm{H})\end{array}$ & $\begin{array}{c}6.48(\mathrm{~s} \\
1 \mathrm{H})\end{array}$ & $\begin{array}{c}6.47(\mathrm{~s} \\
1 \mathrm{H})\end{array}$ & $\begin{array}{c}6.61(\mathrm{~s} \\
1 \mathrm{H})\end{array}$ \\
\hline & 2.58 & 2.49 & 2.48 & 2.47 & 2.47 & 2.47 & 2.47 & 2.46 & 2.43 & 2.39 & 2.67 \\
\hline 7 & $\begin{array}{c}(\mathrm{d}, 1 \mathrm{H}, \\
3.5)\end{array}$ & $\begin{array}{c}(\mathrm{d}, 1 \mathrm{H}, \\
3.5)\end{array}$ & $\begin{array}{c}(\mathrm{d}, 1 \mathrm{H}, \\
3.5)\end{array}$ & $\begin{array}{c}(\mathrm{d}, 1 \mathrm{H}, \\
3.5)\end{array}$ & $\begin{array}{c}(\mathrm{d}, 1 \mathrm{H}, \\
3.5)\end{array}$ & $\begin{array}{c}(\mathrm{d}, 1 \mathrm{H}, \\
3.5)\end{array}$ & $\begin{array}{c}(\mathrm{d}, 1 \mathrm{H}, \\
3.5)\end{array}$ & $\begin{array}{c}(\mathrm{d}, 1 \mathrm{H}, \\
3.5)\end{array}$ & $\begin{array}{c}(\mathrm{d}, 1 \mathrm{H}, \\
3.5)\end{array}$ & $\begin{array}{c}(\mathrm{d}, 1 \mathrm{H}, \\
3.5)\end{array}$ & $\begin{array}{c}(\mathrm{d}, 1 \mathrm{H}, \\
3.5)\end{array}$ \\
\hline & 5.62 & 5.77 & 5.78 & 5.79 & 5.78 & 5.79 & 5.78 & 5.79 & 5.81 & 5.76 & 5.93 \\
\hline 8 & $\begin{array}{l}(\mathrm{dd}, 1 \mathrm{H}, \\
9.5,3.5)\end{array}$ & $\begin{array}{l}(\mathrm{dd}, 1 \mathrm{H}, \\
9.5,3.5)\end{array}$ & $\begin{array}{l}(\mathrm{dd}, 1 \mathrm{H}, \\
9.5,3.5)\end{array}$ & $\begin{array}{l}(\mathrm{dd}, 1 \mathrm{H}, \\
9.5,3.5)\end{array}$ & $\begin{array}{l}(\mathrm{dd}, 1 \mathrm{H}, \\
9.5,3.5)\end{array}$ & $\begin{array}{l}(\mathrm{dd}, 1 \mathrm{H}, \\
9.5,3.5)\end{array}$ & $\begin{array}{l}(\mathrm{dd}, 1 \mathrm{H}, \\
9.5,3.5)\end{array}$ & $\begin{array}{l}(\mathrm{dd}, 1 \mathrm{H}, \\
9.5,3.5)\end{array}$ & $\begin{array}{l}(\mathrm{dd}, 1 \mathrm{H}, \\
9.5,3.5)\end{array}$ & $\begin{array}{l}(\mathrm{dd}, 1 \mathrm{H}, \\
9.5,3.5)\end{array}$ & $\begin{array}{l}(\mathrm{dd}, 1 \mathrm{H}, \\
9.5,3.5)\end{array}$ \\
\hline 9 & $\begin{array}{c}6.06 \\
(\mathrm{~d}, 1 \mathrm{H} \\
9.5)\end{array}$ & $\begin{array}{c}6.03 \\
(\mathrm{~d}, 1 \mathrm{H} \\
9.5)\end{array}$ & $\begin{array}{c}6.04 \\
(\mathrm{~d}, 1 \mathrm{H} \\
9.5)\end{array}$ & $\begin{array}{c}6.04 \\
(\mathrm{~d}, 1 \mathrm{H} \\
9.5)\end{array}$ & $\begin{array}{c}6.04 \\
(\mathrm{~d}, 1 \mathrm{H} \\
9.5)\end{array}$ & $\begin{array}{c}6.03 \\
(\mathrm{~d}, 1 \mathrm{H} \\
9.5)\end{array}$ & $\begin{array}{c}6.03 \\
(\mathrm{~d}, 1 \mathrm{H} \\
9.5)\end{array}$ & $\begin{array}{c}6.04 \\
(\mathrm{~d}, 1 \mathrm{H} \\
9.5)\end{array}$ & $\begin{array}{c}6.03 \\
(\mathrm{~d}, 1 \mathrm{H} \\
9.5)\end{array}$ & $\begin{array}{c}6.02 \\
(\mathrm{~d}, 1 \mathrm{H} \\
9.5)\end{array}$ & $\begin{array}{c}6.09 \\
(\mathrm{~d}, 1 \mathrm{H} \\
9.5)\end{array}$ \\
\hline 12 & $\begin{array}{c}4.87, \\
4.65 \\
(\mathrm{Abq}, \\
2 \mathrm{H}, \\
13.5)\end{array}$ & $\begin{array}{c}4.90, \\
4.65 \\
(\mathrm{Abq}, \\
2 \mathrm{H}, \\
13.5)\end{array}$ & $\begin{array}{c}4.90, \\
4.65 \\
(\mathrm{Abq}, \\
2 \mathrm{H}, \\
13.5)\end{array}$ & $\begin{array}{c}4.90, \\
4.64 \\
(\mathrm{Abq}, \\
2 \mathrm{H}, \\
13.5)\end{array}$ & $\begin{array}{c}4.90,4.64 \\
(\mathrm{Abq}, 2 \mathrm{H}, \\
13.5)\end{array}$ & $\begin{array}{c}4.90, \\
4.64 \\
(\mathrm{Abq}, \\
2 \mathrm{H}, \\
13.5)\end{array}$ & $\begin{array}{c}4.890, \\
4.64 \\
(\mathrm{Abq}, \\
2 \mathrm{H}, \\
13.5)\end{array}$ & $\begin{array}{c}4.89 \\
4.65 \\
(\mathrm{Abq}, \\
2 \mathrm{H}, \\
13.5)\end{array}$ & $\begin{array}{c}4.91, \\
4.64 \\
(\mathrm{Abq}, \\
2 \mathrm{H}, \\
13.5)\end{array}$ & $\begin{array}{c}4.88, \\
4.64 \\
(\mathrm{Abq}, \\
2 \mathrm{H}, \\
13.5)\end{array}$ & $\begin{array}{c}4.93 \\
4.70 \\
(\mathrm{Abq}, \\
2 \mathrm{H}, \\
13.5)\end{array}$ \\
\hline 13 & $\begin{array}{c}1.77(\mathrm{~s}, \\
3 \mathrm{H})\end{array}$ & $\begin{array}{c}1.57(\mathrm{~s}, \\
3 \mathrm{H})\end{array}$ & $\begin{array}{c}1.57(\mathrm{~s}, \\
3 \mathrm{H})\end{array}$ & $\begin{array}{c}1.73(\mathrm{~s}, \\
3 \mathrm{H})\end{array}$ & $\begin{array}{c}1.73(\mathrm{~s}, \\
3 \mathrm{H})\end{array}$ & $\begin{array}{c}1.74(\mathrm{~s}, \\
3 \mathrm{H})\end{array}$ & $\begin{array}{c}1.72(\mathrm{~s}, \\
3 \mathrm{H})\end{array}$ & $\begin{array}{c}1.72(\mathrm{~s}, \\
3 \mathrm{H})\end{array}$ & $\begin{array}{c}1.72(\mathrm{~s}, \\
3 \mathrm{H})\end{array}$ & $\begin{array}{c}1.69(\mathrm{~s}, \\
3 \mathrm{H})\end{array}$ & $\begin{array}{c}1.75(\mathrm{~s}, \\
3 \mathrm{H})\end{array}$ \\
\hline 14 & $\begin{array}{c}1.72(\mathrm{~s}, \\
3 \mathrm{H})\end{array}$ & $\begin{array}{c}1.72(\mathrm{~s}, \\
3 \mathrm{H})\end{array}$ & $\begin{array}{c}1.72(\mathrm{~s}, \\
3 \mathrm{H})\end{array}$ & $\begin{array}{c}1.57(\mathrm{~s}, \\
3 \mathrm{H})\end{array}$ & $\begin{array}{c}1.57(\mathrm{~s}, \\
3 \mathrm{H}))\end{array}$ & $\begin{array}{c}1.56(\mathrm{~s}, \\
3 \mathrm{H})\end{array}$ & $\begin{array}{c}1.56(\mathrm{~s}, \\
3 \mathrm{H})\end{array}$ & $\begin{array}{c}1.58(\mathrm{~s}, \\
3 \mathrm{H})\end{array}$ & $\begin{array}{c}1.58(\mathrm{~s}, \\
3 \mathrm{H})\end{array}$ & $\begin{array}{c}1.47(\mathrm{~s}, \\
3 \mathrm{H})\end{array}$ & $\begin{array}{c}1.59(\mathrm{~s} \\
3 \mathrm{H})\end{array}$ \\
\hline 15 & $\begin{array}{c}1.61(\mathrm{~s}, \\
3 \mathrm{H})\end{array}$ & $\begin{array}{c}1.50(\mathrm{~s}, \\
3 \mathrm{H})\end{array}$ & $\begin{array}{c}1.49(\mathrm{~s}, \\
3 \mathrm{H})\end{array}$ & $\begin{array}{c}1.49(\mathrm{~s}, \\
3 \mathrm{H})\end{array}$ & $\begin{array}{c}1.49(\mathrm{~s}, \\
3 \mathrm{H})\end{array}$ & $\begin{array}{c}1.49(\mathrm{~s} \\
3 \mathrm{H})\end{array}$ & $\begin{array}{c}1.49(\mathrm{~s}, \\
3 \mathrm{H})\end{array}$ & $\begin{array}{c}1.49(\mathrm{~s}, \\
3 \mathrm{H})\end{array}$ & $\begin{array}{c}1.51(\mathrm{~s}, \\
3 \mathrm{H})\end{array}$ & $\begin{array}{c}1.42(\mathrm{~s}, \\
3 \mathrm{H})\end{array}$ & $\begin{array}{c}1.53(\mathrm{~s}, \\
3 \mathrm{H})\end{array}$ \\
\hline
\end{tabular}




\subsection{Insecticidal Activities}

From the insecticidal activities (according to the Table 4), screening, compounds $\mathbf{1 . 1}$ and $\mathbf{1 . 2}$ exhibited higher activity against $3^{\text {th }}$ instar larvae of Mythimna separate than Celangulin-V, compounds(1.3, 1.4, 1.7 and 1.8) exhibited less potency than Celangulin-V, and compounds 1.5, 1.6, 1.9 and 1.10 even no activity at the tested concentration.

Table 4. Insecticidal activities of Celangulin-V derivatives.

\begin{tabular}{cc}
\hline Compounds & Mortality(\%) \\
\hline Acetone & 0 \\
Celangulin-V & 66.7 \\
1.1 & $\mathbf{7 5 . 0}$ \\
1.2 & $\mathbf{8 3 . 3}$ \\
1.3 & 16.7 \\
1.4 & 33.3 \\
1.5 & 0 \\
1.6 & 0 \\
1.7 & 8.3 \\
1.8 & 8.3 \\
1.9 & 0 \\
1.10 & 0 \\
\hline
\end{tabular}

Note: The concentration is a $10 \mathrm{mg}$ test sample dissolved in $1 \mathrm{~mL}$ acetone.

\section{Experimental}

\subsection{General Experimental Procedures}

Melting points were measured on an electrothermal digital apparatus made in Beijing and were uncorrected. The ${ }^{1} \mathrm{H}-\mathrm{NMR}$ (500 MHz), and ${ }^{13} \mathrm{C}-\mathrm{NMR}$ (125 MHz) were obtained on a Bruker AM-500 FT-NMR spectrometer with $\mathrm{CDCl}_{3}$ as the solvent and TMS as the internal standard. MS were recorded under ESI conditions using a Thermo LCQ Fleet instrument. Infrared spectra were measured on a Nicolet FT-IR-20SX instrument using a potassium bromide (KBr) disk, scanning from 625 to $4000 \mathrm{~cm}^{-1}$. Optical rotation was measured by Rudolph Autopol II. Analytical thin layer chromatography (TLC) was carried out on precoated plates (silica gel), and spots were visualized with $\mathrm{H}_{2} \mathrm{SO}_{4} / \mathrm{EtOH}$. Yields were not optimized. Solvents were dried by standard methods. The title compounds were synthesized under a nitrogen atmosphere.

\subsection{Synthesis of the Derivatives of Celangulin- $V$}

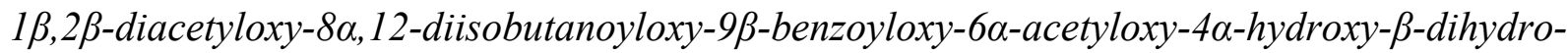
agarofuran (1.1): A mixture of celangulin-V (1) $(0.100 \mathrm{~g}, 0.15 \mathrm{mmol})$ and acetic anhydride $(1 \mathrm{~mL})$ in dry Pyridine (Pyr, $20 \mathrm{~mL}$ ) was stirred over night at room temperature (RT). When the reaction was complete (checked by TLC), $1 \mathrm{~mL}$ methanol was added to quench the reaction, then $50 \mathrm{~mL}$ water was added to the mixture which was then extracted with ethyl acetate $(50 \mathrm{~mL} \times 3)$. The ethyl acetate layers were combined and washed with $30 \mathrm{~mL}$ water and $5 \mathrm{~mL}$ saturated sodium chloride, dried over anhydrous 
sodium sulfate and separated by column chromatography(silica gel, 200 300 mesh) with a gradient of petroleum ether $\left(60-90^{\circ} \mathrm{C}\right)$ and ethyl acetate as eluent to yield compound $\mathbf{1 . 1}$ as white powder.ESI-MS: Found $727[\mathrm{M}+\mathrm{Na}]^{+}$. Its IR spectrum revealed characteristic ester absorptions at $1722 \mathrm{~cm}^{-1}$, and a free hydroxyl absorption at $3552 \mathrm{~cm}^{-1}$. (6-) $\mathrm{OCOCH}_{3}$ was certified by ${ }^{1} \mathrm{H}-\mathrm{NMR} \delta: 2.13(\mathrm{~s}, 3 \mathrm{H})$ and ${ }^{13} \mathrm{C}-\mathrm{NMR}$ $\delta$ : $169.58(\mathrm{CO}), 21.42\left(\mathrm{CH}_{3}\right)$, other data see Table 2 and Table 3.

\section{1 $\beta, 2 \beta$-diacetyloxy-8 $\alpha, 12$-diisobutanoyloxy-9 $\beta$-benzoyloxy-6 $\alpha$-propyloxy-4 $\alpha$-hydroxy- $\beta$-dihydro-}

agarofuran (1.2): This compound was obtained as a white powder following a similar procedure to the synthesis of compound 1.1. ESI-MS: Found $741[\mathrm{M}+\mathrm{Na}]^{+}$. Its IR spectrum revealed characteristic ester absorptions at $1726 \mathrm{~cm}^{-1}$, and a free hydroxyl absorption at $3548 \mathrm{~cm}^{-1}$. (6-) $\mathrm{OCOCH}_{2} \mathrm{CH}_{3}$ was certificated by ${ }^{1} \mathrm{H}-\mathrm{NMR} \delta: 1.19(3 \mathrm{H}, \mathrm{t}, 7.5), 2.42(2 \mathrm{H}, \mathrm{q}, 7.5)$ and ${ }^{13} \mathrm{C}-\mathrm{NMR} \delta: 172.84(\mathrm{CO}), 28.21 \mathrm{CH}_{2}$, $8.95 \mathrm{CH}_{3}$, other data see Table 2 and Table 3.

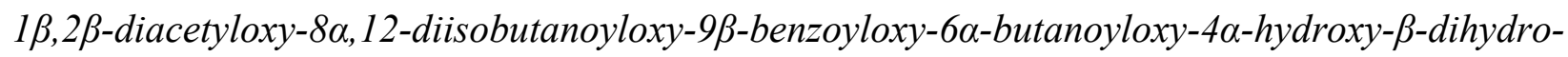

agarofuran (1.3): This compound was obtained as a white powder following a similar procedure to the synthesis of the compound 1.1. ESI-MS: Found $755[\mathrm{M}+\mathrm{Na}]^{+}$. Its IR spectrum revealed characteristic ester absorptions at $1721 \mathrm{~cm}^{-1}$, and a free hydroxyl absorption at $3546 \mathrm{~cm}^{-1}$. (6-)OCOCH $\mathrm{CH}_{2} \mathrm{CH}_{3}$ was certificated by ${ }^{1} \mathrm{H}-\mathrm{NMR} \delta: 2.35(\mathrm{~m}, 2 \mathrm{H}), 1.70(\mathrm{~m}, 2 \mathrm{H}), 0.96(3 \mathrm{H}, \mathrm{t}, 7.0)$ and ${ }^{13} \mathrm{C}-\mathrm{NMR} \delta: 172.07(\mathrm{CO})$, 18.16 $\mathrm{CH}_{2}, 36.75 \mathrm{CH}_{2}, 13.73 \mathrm{CH}_{3}$, other data see Table 2 and Table 3.

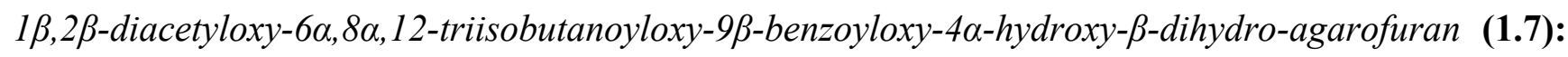
This compound was obtained as a white powder following a similar procedure to the synthesis of the compound 1.1. ESI-MS: Found $755[\mathrm{M}+\mathrm{Na}]^{+}$. Its IR spectrum revealed characteristic ester absorptions at $1724 \mathrm{~cm}^{-1}$, and a free hydroxyl absorption at $3542 \mathrm{~cm}^{-1}$. (6-) $\mathrm{OCOCH}\left(\mathrm{CH}_{3}\right)_{2}$ was certificated by ${ }^{1} \mathrm{H}-\mathrm{NMR} \delta: 1.21(\mathrm{~d}, 3 \mathrm{H}, 7.0), 1.22(\mathrm{~d}, 3 \mathrm{H}, 7.0), 2.59(\mathrm{~m}, 1 \mathrm{H})$ and ${ }^{13} \mathrm{C}-\mathrm{NMR} \delta: 177.00(\mathrm{CO}), 34.42 \mathrm{CH}$, $18.99 \mathrm{CH}_{3}, 19.14 \mathrm{CH}_{3}$, other data see Table 2 and Table 3.

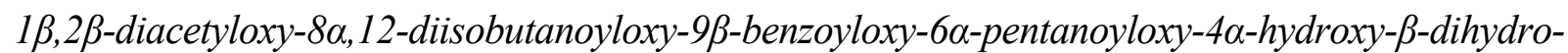

agarofuran (1.4): A mixture of celangulin-V (1) $(0.100 \mathrm{~g}, 0.15 \mathrm{mmol})$, pentanoic acid (1 mL), N,N-dicyclohexylcarbodiimide (DCC, $0.062 \mathrm{~g}, 0.30 \mathrm{mmol}$ ) and dimethylaminopyridine (DMAP, $0.005 \mathrm{~g}, 0.04 \mathrm{mmol}$ ) in dry dichloromethane (DCM $20 \mathrm{~mL}$ ) was stirred over night at room temperature. When the reaction was complete (checked by TLC), $1 \mathrm{~mL}$ methanol was added to quench the reaction. Then $30 \mathrm{~mL}$ water was added to the mixture and the resulting suspension was filtered then extracted with dichloromethane $(30 \mathrm{~mL} \times 3)$. The dichloromethane layers were combined and washed with $30 \mathrm{~mL}$ water and $5 \mathrm{~mL}$ saturated sodium chloride, dried over anhydrous sodium sulfate and separated by column chromatography(silica gel, 200 300 mesh) with a gradient of petroleum ether $\left(60-90{ }^{\circ} \mathrm{C}\right)$ and ethyl acetate as eluent to yield compound $\mathbf{1 . 4}$ as a white powder. ESI-MS: Found 769 $[\mathrm{M}+\mathrm{Na}]^{+}$. Its IR spectrum revealed characteristic ester absorptions at $1718 \mathrm{~cm}^{-1}$, and a free hydroxyl absorption at $3547 \mathrm{~cm}^{-1}$. (6-) $\mathrm{OCOCH}_{2} \mathrm{CH}_{2} \mathrm{CH}_{2} \mathrm{CH}_{3}$ was certificated by ${ }^{1} \mathrm{H}-\mathrm{NMR} \delta: 0.92(\mathrm{~m}, 3 \mathrm{H}), 1.33$ $(\mathrm{m}, 2 \mathrm{H}), 2.38(\mathrm{~m}, 2 \mathrm{H}), 1.65(\mathrm{~m}, 2 \mathrm{H})$ and ${ }^{13} \mathrm{C}-\mathrm{NMR} \delta: 172.24(\mathrm{CO}), 13.66 \mathrm{CH}_{3}, 22.27 \mathrm{CH}_{2}, 26.68 \mathrm{CH}_{2}$, $34.58 \mathrm{CH}_{2}$, other data see Table 2 and Table 3. 


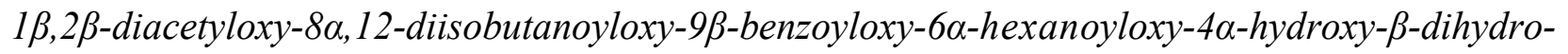
agarofuran (1.5): This compound was obtained as a white powder following a similar procedure to the synthesis of the compound 1.4. ESI-MS: Found $783[\mathrm{M}+\mathrm{Na}]^{+}$. Its IR spectrum revealed characteristic ester absorptions at $1718 \mathrm{~cm}^{-1}$, and a free hydroxyl absorption at $3543 \mathrm{~cm}^{-1}$. (6-) $\mathrm{OCOCH}_{2} \mathrm{CH}_{2} \mathrm{CH}_{2} \mathrm{CH}_{2} \mathrm{CH}_{3}$ was certificated by ${ }^{1} \mathrm{H}-\mathrm{NMR} \delta: 0.88(\mathrm{t}, 3 \mathrm{H}, 7.0), 1.31(\mathrm{~m}, 4 \mathrm{H}), 2.38$ (m, 2H), $1.68(\mathrm{~m}, 2 \mathrm{H})$ and ${ }^{13} \mathrm{C}-\mathrm{NMR} \delta: 172.07(\mathrm{CO}), 22.24 \mathrm{CH}_{2}, 24.31 \mathrm{CH}_{2}, 31.26 \mathrm{CH}_{2}, 34.81 \mathrm{CH}_{2}, 13.87$ $\mathrm{CH}_{3}$, other data see Table 2 and Table 3.

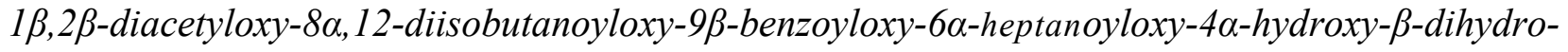

agarofuran (1.6): This compound was obtained as a colorless oil following a similar procedure to the synthesis of the compound 1.4.ESI-MS: Found $797[\mathrm{M}+\mathrm{Na}]^{+}$. Its IR spectrum revealed characteristic ester absorptions at $1716 \mathrm{~cm}^{-1}$, and a free hydroxyl absorption at $3547 \mathrm{~cm}^{-1}$. (6-) $\mathrm{OCOCH}_{2} \mathrm{CH}_{2} \mathrm{CH}_{2} \mathrm{CH}_{2} \mathrm{CH}_{2} \mathrm{CH}_{3}$ was certificated by ${ }^{1} \mathrm{H}-\mathrm{NMR} \delta: 0.88(\mathrm{t}, 3 \mathrm{H}, 7.0), 1.29(\mathrm{~m}, 6 \mathrm{H})$, $2.36(\mathrm{~m}, 2 \mathrm{H}), 1.66(\mathrm{~m}, 2 \mathrm{H})$ and ${ }^{13} \mathrm{C}-\mathrm{NMR} \delta: 172.26(\mathrm{CO}), 22.47 \mathrm{CH}_{2}, 24.61 \mathrm{CH}_{2}, 28.82 \mathrm{CH}_{2}, 31.37 \mathrm{CH}_{2}$, $34.86 \mathrm{CH}_{2}, 14.01 \mathrm{CH}_{3}$, other data see Table 2 and Table 3.

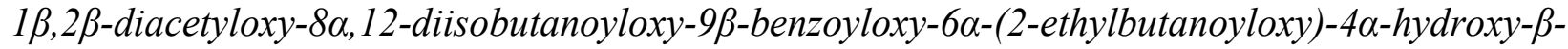

dihydro-agarofuran (1.8): This compound was obtained as a white powder following a similar procedure to the synthesis of the compound 1.4. ESI-MS: Found $783[\mathrm{M}+\mathrm{Na}]^{+}$. Its IR spectrum revealed characteristic ester absorptions at $1720 \mathrm{~cm}^{-1}$, and a free hydroxyl absorption at $3544 \mathrm{~cm}^{-1}$. (6-) $\mathrm{OCOCH}\left(\mathrm{CH}_{2} \mathrm{CH}_{3}\right)_{2}$ was certificated by ${ }^{1} \mathrm{H}-\mathrm{NMR} \delta: 2.28(1 \mathrm{H}, \mathrm{m}), 1.70(4 \mathrm{H}, \mathrm{m}), 0.91(6 \mathrm{H}, \mathrm{m})$ and ${ }^{13} \mathrm{C}-\mathrm{NMR} \delta$ : $174.66(\mathrm{CO}), 49.29 \mathrm{CH}, 24.58 \mathrm{CH}_{2}, 24.25 \mathrm{CH}_{2}, 12.02 \mathrm{CH}_{3}, 11.88 \mathrm{CH}_{3}$, other data see Table 2 and Table 3.

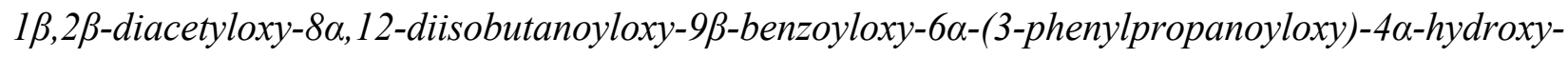
$\beta$-dihydro-agarofuran (1.9): This compound was obtained as a white powder following a similar procedure to the synthesis of the compound 1.4. ESI-MS: Found $817[\mathrm{M}+\mathrm{Na}]^{+}$. Its IR spectrum revealed characteristic ester absorptions at $1716 \mathrm{~cm}^{-1}$, and a free hydroxyl absorption at $3547 \mathrm{~cm}^{-1}$. (6-) $\mathrm{OCOCH}_{2} \mathrm{CH}_{2} \mathrm{C}_{6} \mathrm{H}_{5}$ was certificated by ${ }^{1} \mathrm{H}-\mathrm{NMR} \delta$ : $2.72(\mathrm{~m}, 2 \mathrm{H}), 3.00(\mathrm{~m}, 2 \mathrm{H}), 7.29(\mathrm{~m}, 2 \mathrm{H}), 7.20$ $(\mathrm{m}, 3 \mathrm{H})$ and ${ }^{13} \mathrm{C}-\mathrm{NMR} \delta$ : $171.45(\mathrm{CO}), 36.36 \mathrm{CH}_{2}, 30.68 \mathrm{CH}_{3}, 140.05 \mathrm{C}, 126.41 \mathrm{CH}, 128.57$ $\mathrm{CH} \times 2,128.31 \mathrm{CH} \times 2$, other data see Table 2 and Table 3 .

\section{1 $\beta, 2 \beta$-diacetyloxy- $8 \alpha, 12$-diisobutanoyloxy- $6 \alpha, 9 \beta$-dibenzoyloxy-4 $\alpha$-hydroxy- $\beta$-dihydro-}

agarofuran (1.10): This compound was obtained as a white powder following a similar procedure to the synthesis of the compound 1.4. ESI-MS: Found $789[\mathrm{M}+\mathrm{Na}]^{+}$. Its IR spectrum revealed characteristic ester absorptions at $1722 \mathrm{~cm}^{-1}$, and a free hydroxyl absorption at $3543 \mathrm{~cm}^{-1}$. (6-) $\mathrm{OCOC}_{6} \mathrm{H}_{5}$ was certificated by ${ }^{1} \mathrm{H}-\mathrm{NMR} \delta: 8.19(2 \mathrm{H}, \mathrm{m}), 7.47(2 \mathrm{H}, \mathrm{m}), 7.58(1 \mathrm{H}, \mathrm{m})$ and ${ }^{13} \mathrm{C}-\mathrm{NMR} \delta: 165.48(\mathrm{CO})$, $128.67(2 \times \mathrm{CH}), 133.46 \mathrm{CH}, 130.18(2 \times \mathrm{CH}), 129.64 \mathrm{C}$, other data see Table 2 and Table 3 .

\subsection{Bioassay for Insecticidal Activities}

Wheat leaf discs of known area $(0.5 \mathrm{~cm} \times 0.5 \mathrm{~cm})$ were treated with $1 \mu \mathrm{L}$ from $10 \mathrm{mg}$ test samples dissolved in $1 \mathrm{~mL}$ acetone (acetone and celangulin $\mathrm{V}$ were used as negative and positive controls). The 
$3^{\text {rd }}$ instar larvae of Mythimna separate were fed with the discs over $2 \mathrm{~h}$ (repeated 3 times for each sample). After $2 \mathrm{~h}$, the numbers of knocked down larvae (symptoms: the larvae were narcotized, the bodies were very soft and immobilized, and response disappeared completely) were recorded. [3].The insecticidal activity result can be found in Table 4 .

\section{Conclusions}

In conclusion, ten new 6-acyloxy derivatives of Celangulin-V (1.1-1.10) have been synthesized and evaluated for their insecticidal activities against the $3^{\text {rd }}$ instar larvae of Mythimna separate in vivo at a concentration of $10 \mathrm{mg} \cdot \mathrm{mL}^{-1}$. The bioassay of these analogues showed that some synthesized ester-derivatives of Celangulin-V showed the same stomach toxic effects against Mythimna separate as Celangulin- $\mathrm{V}$ and that the poisoned insects had the same symptoms as from its ether-derivatives. This implies the insecticidal ester-derivatives may act on the same target protein as Celangulin-V. The substitutions of C-6 have a notable influence on the insecticidal activity. Among the Celangulin-V ester-derivatives, two and three carbon substitutions may be more favorable for promoting the insecticidal activity as compared with much larger substitution groups at the C-6 position. These results have encouraged us to start further investigations into novel Celangulin- $\mathrm{V}$ derivatives as insecticidal agents and this will be reported in due course.

\section{Acknowledgements}

This work was supported by the National Key S\&T Research Foundation of China (2010CB126105) and the National Natural Science Foundation of China $(30871663,30800729)$ as well as the program for Excellent Young Talents in Northwest A\&F University (QNGG-2009-020) and the Funds of Central Colleges Basic Scientific Research Operating Expenses(QN2011117).

\section{References}

1. Gao, J.M.; Wu, W.J.; Zhang, J.W.; Konishi, Y. The dihydro- $\beta$-agarofuran sesquiterpenoids. Nat. Prod. Rep. 2007, 24, 1153-1189.

2. Spivey, A.C.; Weston, M.; Woodhead, S. Celastraceae sesquiterpenoids: Biological activity and synthesis. Chem. Soc. Rev. 2002, 31, 43-59.

3. Wei, S.P.; Wang, M.A.; Zhang, J.W.; Qian, Y.; Ji, Z.Q.; Wu, W.J. Three novel sesquiterpene esters from Celastrus angulatus. Nat. Prod. Commun. 2009, 4, 461-466.

4. Wu, W.J.; Tu, Y.Q.; Liu, H.X.; Zhu, J.B. Celangulin II, III and IV: New insecticidal sesquiterpenoids from Celastrus angulatus. J. Nat. Prod. 1992, 55, 1294-1298.

5. Wang, M.T.; Qin, H.L.; Kong, M.; Li, Y.Z. Insecticidal sesquiterpene polyol ester from Celastrus angulatus. Phytochemistry 1991, 30, 3931-3933.

6. Wu, W.J.; Li, S.B.; Zhu, J.B.; Liu, H.X. New Sesquiterpenoid celangulin V: Isolation and determination. J. Northwest A\&F Univ. 1994, 22, 116-117.

7. Qi, Z.J.; Xue, X.P.; Wu, W.J.; Zhang, J.W.; Yang, R.Y. Preparation of monoclonal antibody against celangulin V and immunolocalization of receptor in the oriental armyworm, Mythimna separata walker (Lepidoptera: Noctuidae). J. Agric. Food Chem. 2006, 54, 7600-7605. 
8. Zhang, J.W.; Hu, Z.N.; Yang, H.; Wu, W.J. Synthesis and insecticidal activities of new ether-derivatives of celangulin-V. Nat. Prod. Commun. 2010, 5, 845-848.

(C) 2011 by the authors; licensee MDPI, Basel, Switzerland. This article is an open access article distributed under the terms and conditions of the Creative Commons Attribution license (http://creativecommons.org/licenses/by/3.0/). 